\title{
RELATION ENTRE L'AMPLITUDE ET LA DUREE DU CYCLE POUR LES NOVAE RECURRENTES ET LES VARIABLES DU TYPE U GEMINORUM
}

\author{
A. N. ESKİŏLLU * \\ (Observetoire de l'Université d'Ankara, Ankara)
}

\section{$\ddot{\mathbf{O z e t}}$}

Amplitüd ile sikl arasındaki bağıntı ilk defa Kukarkin ve Parenago tarafindan yayınlanmıștı. Bugün kesin olarak bilinen $\mathbf{U}$ Geminorum yldızlarının sayısı eskiye nazaran daha fazla olduğundan, son bilinen duruma göre bu bağıntının yeni bir şekli kurulmuştur.

\section{Sommaire}

Une relation entre l'amplitude et la durée du cycle a été publiée, pour la première fois par Kukarkin et Parenago. Comme le nobre des étoiles U Geminorum connues aujourd'hui avec précision est plus grand qu' autrefois, une nouvelle forme de cette relation a été établie ave les données récentes.

Les intervalles entre les explosions des novae recurrentes ne semblent pas montrer de périodicité bien définie. Tout récemment, M. Friedjung [1] a cependant tenté de mettre en évidence une période fondamentale. Il a trouvé une valeur de 6 ans environ.

B.V. Kukarkin et P.P. Parenago [2] ont trouvé d'autre part une corrélation entre l'amplitude et les intervalles entre les explosions pour les étoiles U Geminorum, corrélation qu'ils ont étendue aux novae récurrentes connues en 1934. Ils ont abouti à la relation suivante entre l'amplitude et le cycle moyen;

\footnotetext{
* Adresse : Fen Fakültesi, Ankara.
} 
$A=0,63+1,667 \log P$, (Ici $A$ indique l'amplitude et $P$ la période exprimée en jours). Comme le nombre des étoiles $U$ Geminorum est augmenté depuis la date cité, j'ai revu cette question à l'aide des étoiles du type $U$ Geminorum dont les amplitudes sont connues et des novae récurrentes [3]. Les minimums de quelques étoiles U Gem ont été tirés du travail de V. A. Vorobyeva [4] qui les a estimés sur les cartes du Sky Atlas. Finalement j'ai pu réunir les données nécessaires pour 44 étoiles $U$ Geminorum et 5 novaé récurrentes. Elles figurent dans le tableau inclu. Les cycles moyens sont donnés en jours. La relation trouvée par la méthode des moindres carrés est la suivante :

$$
\mathrm{A}=1,23+1,56 \log \mathbf{P}
$$

dont le terme constant différe notablement de celui de Kukarkin et Parenago. La droite correspondante est représentée dans la figure qui donne pour chaque étoile l'amplitude et le logarithme du cycle moyen. Une telle relation résulte peut-etre d'une parenté entre les novae récurrentes et les variables du type $U$ Geminorum. Plusieus de ces derniéres sont d'ailleurs des binaires comme le sont certaines novae récurrentes ou non. 


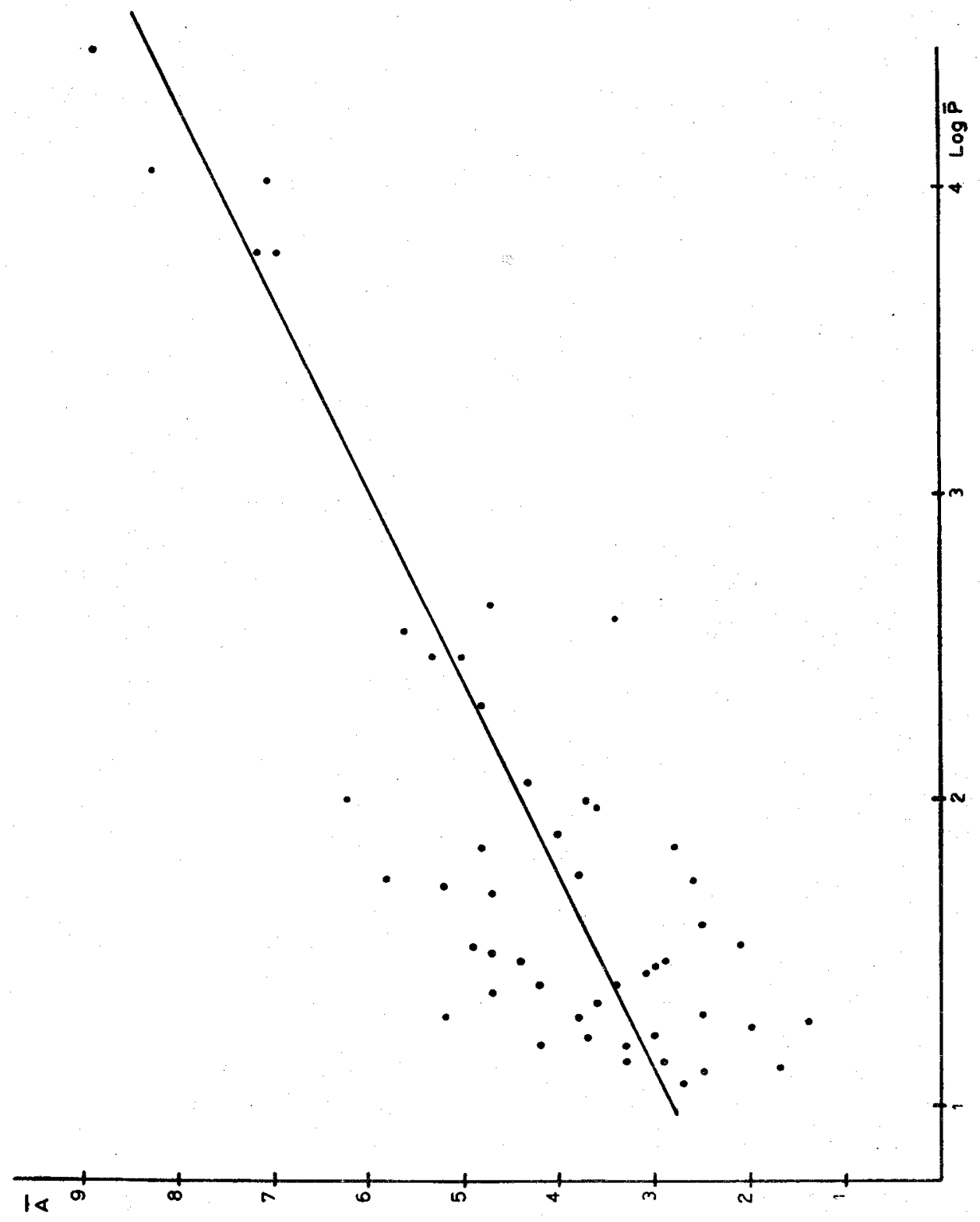




\section{TABLEAU}

Amplitudes (A) et cycles (P) des variables du type U Geminorum

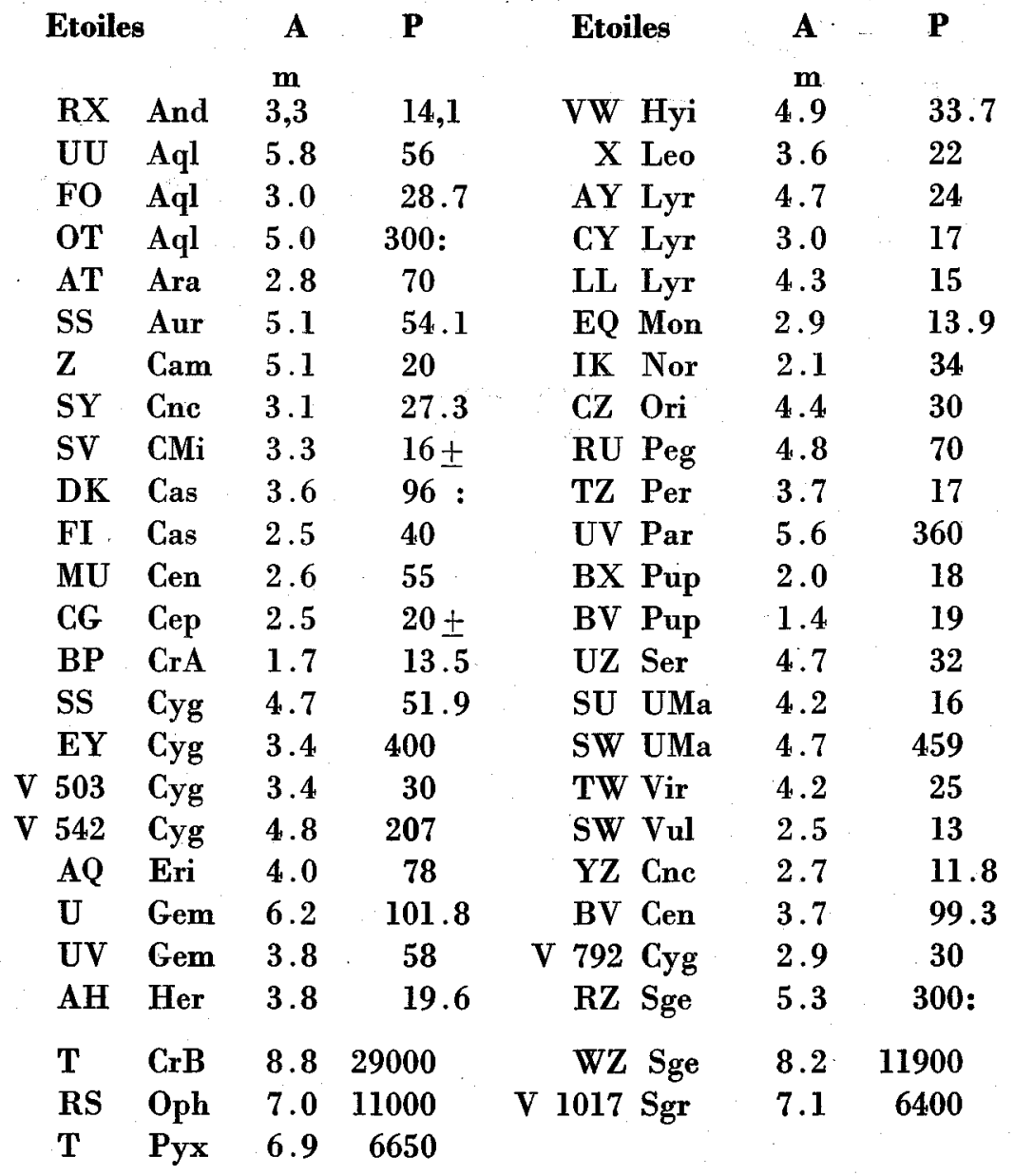




\section{Bibliographie}

[1] M. Friedjung, J. Brit. Astron. Ass. 72,276 (1962)

[2] B.V. Kukarkin and P.P. Parenago, Variable Stars, 4,251 (1934)

[3] A.N. Eskioğlu, Ann. Astrophys. 26, 331 (1963)

[4] V.A. Vorobyeva, Peremm. Zvezdy, 13, 72 (1960)

(Reçu le 8 décembre 1965)

Fin du Tome XIV

de la Série $A$ 



\section{Communications de la Faculté des Sciences de I'Université d'Ankara Série A, Tome XIV}

\section{Table des matiéres}

Pervin Yazgan : Results on some plane nets ........ 1 Z. Güner : The temperature dispersion of complex dielectric constant of dipolar liquids in the microwave region $\lambda=3.2 \mathrm{~cm} .(9375 \mathrm{Mc} / \mathrm{s}) \ldots \ldots \ldots \ldots \ldots \ldots \ldots .6 . \ldots \ldots$

A. N. Eskioğlu : Relation entre l'amplitude et la durée du cycle pour les novae recurrentes et les variables du type $\mathrm{U}$ geminorum $\ldots \ldots \ldots \ldots \ldots \ldots \ldots \ldots \ldots . . . . .$. 